\title{
Asymmetry of Unipolar Electrograms in a Thin Tissue with Epicardial-Endocardial Activation Delay
}

\author{
Eric Irakoze ${ }^{1,2}$, Chirasvi Halekote Ramesh Gowda ${ }^{1,2}$, Vincent Jacquemet ${ }^{1,2}$ \\ ${ }^{1}$ Université de Montréal, Montreal, Canada \\ ${ }^{2}$ Hôpital du Sacré-Cœur, Montreal, Canada
}

\begin{abstract}
Experimental evidences suggest the occurrence of epicardial-endocardial activation delays in structurally remodeled atrial tissue. Our aim was to investigate the consequences of this delay on electrogram morphology.

We created a $50 \times 50 \times 2 \mathrm{~mm}$ monodomain model of atrial tissue composed of an epicardial and an endocardial layer (1 mm thickness each) with different conductivities. Plane waves with epicardial-endocardial delays were simulated. Unipolar electrograms were computed. Simulated electrogram asymmetry was compared to a theoretical formula that expresses asymmetry in terms of the angle between the equivalent dipole and the tissue surface.

The results showed that electrogram asymmetry is strongly related to the sine of the angle of the equivalent dipole. Further analysis suggests that epicardialendocardial delay and transmural coupling are important determinants of asymmetry. These findings contribute to the interpretation of unipolar electrogram morphology.
\end{abstract}

\section{Introduction}

Atrial unipolar electrograms can be recorded during clinical interventions such as electrophysiological studies or catheter ablation of atrial arrhythmia. The morphology of these signals may be used to guide the procedure [1]. Asymmetry of unipolar electrogram waveforms has been proposed as a morphology parameter. It is related to the ratio of the amplitude of the positive deflection ( $\mathrm{R}$ wave) to that of the negative deflection ( $\mathrm{S}$ wave) [1].

The determinants of electrogram asymmetry include wavefront curvature, wavefront collisions, anisotropy and conduction heterogeneity [3]. Predominance of waveforms with larger $\mathrm{S}$ waves has been reported in atrial fibrillation patients [2]. Houben et al. postulated that the observed asymmetry reflected differences in conduction between the epicardial and the endocardial layers [2].

Experimental evidences confirmed the existence of an epicardial-endocardial activation delay in structurally re- modeled atrial tissue $[4,5]$. To simulate the effect of transmural activation, bilayer models of atrial tissue have been developed [6,7].

In this paper, we propose to help the interpretation of electrogram asymmetry in the presence of epicardialendocardial delay through a simple model of equivalent dipole.

\section{Methods}

\subsection{Theory}

Using volume conduction theory, the morphology of electrograms generated by a moving line of distributed dipoles will be derived analytically.

A line of dipoles positioned along the $y$ axis propagates in the $x$ direction: $\mathbf{r}_{\text {dipole }}=(x(t), y, 0)$ where the function $x(t)$ describes the motion of the line of dipoles. The linear density of dipole $\mathbf{p}$ is uniform and lies in the $x$ - $z$ plane: $\mathbf{p}=\left(p_{x}, 0, p_{z}\right)$. The measuring electrode is located on the $z$ axis: $\mathbf{r}_{\text {elec }}=(0,0, z)$. The vector from the source to the electrode is therefore $\mathbf{r}=\mathbf{r}_{\text {elec }}-\mathbf{r}_{\text {dipole }}=(-x(t),-y, z)$.

In an unbounded uniform isotropic volume conductor of conductivity $\sigma$, the potential generated by a segment of distributed dipoles from $y=-L / 2$ to $y=L / 2$ is

$$
\begin{aligned}
\phi(t) & =\frac{1}{4 \pi \sigma} \int_{-L / 2}^{L / 2} \mathrm{~d} y \frac{\mathbf{r} \cdot \mathbf{p}}{r^{3}} \\
& =\frac{1}{4 \pi \sigma} \cdot \frac{L\left(z p_{z}-x p_{x}\right)}{\left(x^{2}+z^{2}\right) \sqrt{x^{2}+z^{2}+L^{2} / 4}} \\
& \rightarrow \frac{1}{2 \pi \sigma} \cdot \frac{z p_{z}-x p_{x}}{x^{2}+z^{2}}=K \frac{z \kappa-x(t)}{x(t)^{2}+z^{2}}
\end{aligned}
$$

for $L \rightarrow \infty$ with the constants $K=p_{x} / 2 \pi \sigma$ and $\kappa=$ $p_{z} / p_{x}$.

To determine the amplitudes of the positive and negative deflections, $\mathrm{d} \phi / \mathrm{d} t$ is set to zero, assuming $\mathrm{d} x(t) / \mathrm{d} t>$ 0 . There are two extrema: when the line of dipole is at position $x_{ \pm}=z\left(\kappa \pm \sqrt{\kappa^{2}+1}\right)$. The amplitude $R$ of the positive deflection and the amplitude $S$ of the negative 
deflection (in absolute value) are given by

$$
\begin{aligned}
R & =\left|\phi\left(x_{-}\right)\right|=-\frac{K}{2 x_{-}} \\
S & =\left|\phi\left(x_{+}\right)\right|=\frac{K}{2 x_{+}}
\end{aligned}
$$

since $x_{-}<0<x_{+}$. As a result, the amplitude of the electrogram waveform is

$$
A=R+S=\frac{K}{z} \sqrt{\kappa^{2}+1}=\frac{\|\mathbf{p}\|}{2 \pi \sigma z}
$$

and its asymmetry [3] is given by

$$
a=\frac{R-S}{R+S}=\frac{\kappa}{\sqrt{\kappa^{2}+1}}=\sin (\alpha)
$$

where $\alpha$ is the angle between the dipole $\mathbf{p}$ and the plane of propagation.

This idealized model suggests that electrogram amplitude is associated with dipole magnitude while electrogram asymmetry reflects dipole orientation. Note that the asymmetry does not depend on conductivity, propagation velocity and electrode distance.

\subsection{Atrial tissue model}

The relation between dipole source and electrogram morphology was studied in a $50 \times 50 \times 2 \mathrm{~mm}$ monodomain model of atrial tissue with $0.2 \mathrm{~mm}$ discretization. The geometry was composed of two layers $(1 \mathrm{~mm}$ thickness each) representing the epicardium and the endocardium. Conduction properties were specified by three parameters: the conductivity in the epicardial and in the endocardial layers $\left(\sigma_{\text {epi }}\right.$ and $\left.\sigma_{\text {endo }}\right)$, and the transmural conductivity $\left(\sigma_{\mathrm{z}}\right)$. Membrane kinetics was described by a Courtemanche model modified to represent electrically remodeled cells [8].

Plane waves propagating in the $x$ direction were simulated to reproduce the conditions of a moving line of dipoles. Because of translational symmetry in the $y$ axis, the monodomain equation was solved in two dimensions, a $50 \times 2 \mathrm{~mm}$ transmural section of the tissue. Activation times were defined as crossing of the transmembrane potential with $-40 \mathrm{mV}$. From the resulting activation map, the conduction velocity and the activation delay between the epicardium and the endocardium were computed at the center of the tissue $(x=25 \mathrm{~mm})$. By convention, negative delays corresponded to endocardium being activated first.

\subsection{Parameter specification}

Since conduction velocity is a known determinant of electrogram morphology, parameter sets $\left(\sigma_{\text {epi }}, \sigma_{\text {endo }}, \sigma_{\mathrm{z}}\right)$ that result in a conduction velocity of 40,50 or $60 \mathrm{~cm} / \mathrm{s}$

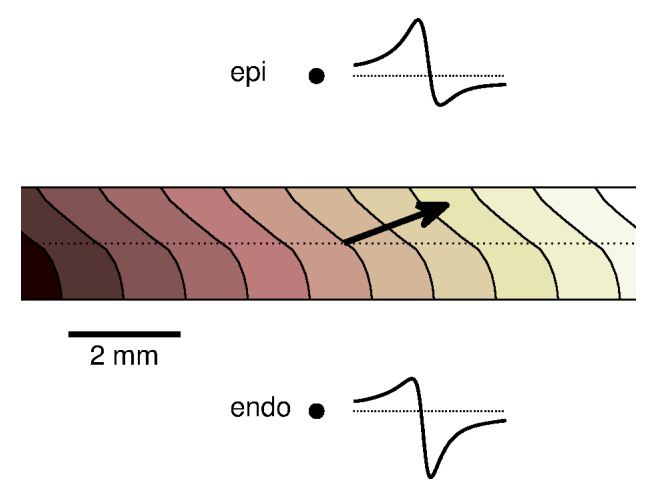

Figure 1. Example of activation map in the central part of the tissue $\left(\sigma_{\text {epi }}=0.77 \mathrm{mS} / \mathrm{cm}, \sigma_{\text {endo }}=2.39 \mathrm{mS} / \mathrm{cm}\right.$, $\left.\sigma_{\mathrm{z}}=1 \mathrm{mS} / \mathrm{cm}\right)$. Electrode positions are shown as a black dot with their corresponding electrogram. The arrow represents the equivalent dipole.

were sought. This enabled the comparison of electrograms in tissues with different parameters but with the same conduction velocity. For that purpose, $\sigma_{\mathrm{z}}$ was varied from 0.5 to $5 \mathrm{mS} / \mathrm{cm}$ and $\sigma_{\text {epi }}$ from 0 to $2.2 \mathrm{mS} / \mathrm{cm}$. The parameter $\sigma_{\text {endo }}$ was iteratively adjusted until the target conduction velocity was reached. The regula falsi zero finding algorithm was used. Each iteration involved simulating plane wave propagation and estimating conduction velocity. A total of 140 parameter sets were obtained for each of the three conduction velocities.

\subsection{Simulated electrograms}

Extracellular potentials were computed assuming an unbounded uniform volume conductor. For each node of the two-dimensional mesh, formula (2) was applied with $L=5 \mathrm{~cm}$ and the current-dipole source obtained from the monodomain model: $p_{x}=-\sigma_{l} \partial V_{m} / \partial x$ and $p_{z}=$ $-\sigma_{\mathrm{z}} \partial V_{m} / \partial z$, where $V_{m}$ is the transmembrane potential, and $\sigma_{l}$ represents either $\sigma_{\text {epi }}$ or $\sigma_{\text {endo. The contributions }}$ from all the nodes to the electrogram were summed up.

Electrograms were computed every $0.1 \mathrm{~ms}$ at the center of the tissue, $2 \mathrm{~mm}$ from the tissue surface on both sides (Fig. 1). Thank to the epi-endo symmetry, the electrogram on the other side was interpreted as coming from the situation with reversed activation delay. Positive $(R)$ and negative $(S)$ deflection amplitudes were measured on each electrogram and the values of $A$ and $a$ were derived from $R$ and $S$.

To make the link with the theoretical formulas (4) and (7), a single value for the equivalent dipole vector was extracted by integrating $p_{x}$ and $p_{z}$ over the monodomain geometry when the wave front was near the tissue center. 


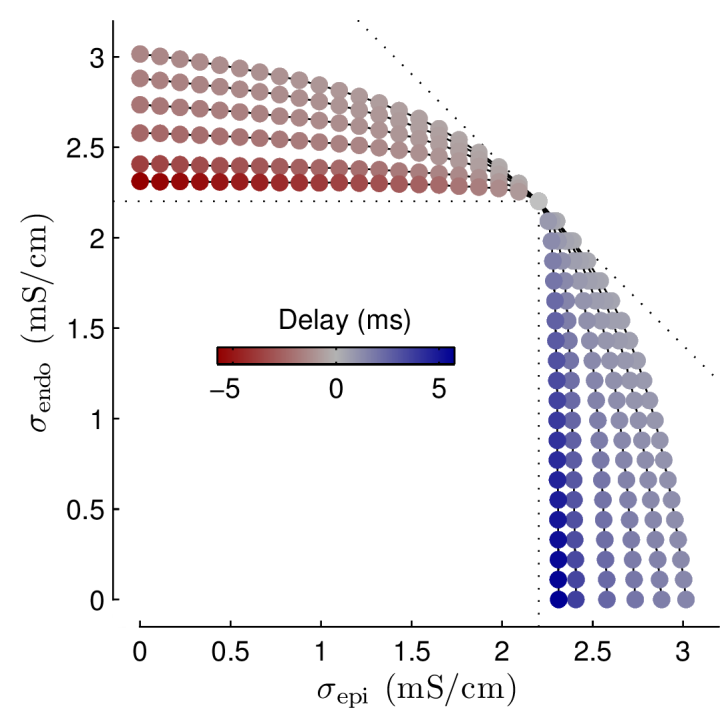

Figure 2. Parameter sets $\left(\sigma_{\text {epi }}, \sigma_{\text {endo }}\right)$ leading to a conduction velocity of $50 \mathrm{~cm} / \mathrm{s}$. The curves correspond to $\sigma_{\mathrm{z}}=0.5,1,2,3,4,5 \mathrm{mS} / \mathrm{cm}$. The dotted square is the limit for $\sigma_{\mathrm{z}} \rightarrow 0$ and the dotted diagonal is the limit for $\sigma_{\mathrm{z}} \rightarrow \infty$. The epi-endo activation delay is color coded. Negative delay means that endo is activated first.

\section{Results}

Figure 2 illustrates the sets of conductivities that led to a conduction velocity of $50 \mathrm{~cm} / \mathrm{s}$. An decrease in $\sigma_{\text {epi }}$ can be compensated by an increase in $\sigma_{\text {endo }}$. When the coupling $\sigma_{\mathrm{z}}$ is stronger, a larger difference between $\sigma_{\text {endo }}$ and $\sigma_{\text {epi }}$ is needed. The curves are symmetric with respect to exchanging the roles of epi and endo. Note that all the tissue activates even if $\sigma_{\text {epi }}=0$ due to transmural propagation from the endocardial layer.

The epi-endo delay (color coded in Fig. 2) ranged between $\pm 5.8 \mathrm{~ms}$ regardless of conduction velocity in the 40 to $60 \mathrm{~cm} / \mathrm{s}$ range. Weaker $\sigma_{\mathrm{z}}$ coupling and larger $\sigma_{\text {epi }}-$

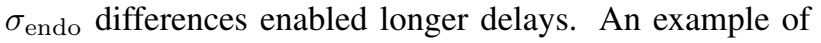
activation map with epi-endo delay is shown in Fig. 1.

Electrogram asymmetry ranged from -0.69 to 0.72 at $40 \mathrm{~cm} / \mathrm{s},-0.64$ to 0.62 at $50 \mathrm{~cm} / \mathrm{s}$ and -0.58 to 0.54 at $60 \mathrm{~cm} / \mathrm{s}$. When the endocardium was activated first, the endocardial electrogram had negative asymmetry and the epicardial electrogram had positive asymmetry, as indicated by the direction of the equivalent dipole (Fig. 1).

Figure 3 demonstrates the correlation between electrogram asymmetry and $\sin (\alpha)$ of the equivalent dipole, thus verifying Eq. (7) in the context of monodomain simulations. The root-mean-square prediction errors were 0.012 , $0.019,0.027$ for a conduction velocity of 40, 50 and $60 \mathrm{~cm} / \mathrm{s}$ respectively. All correlation coefficients were $>0.999$.

Since the angle of the dipole is not available experimen-

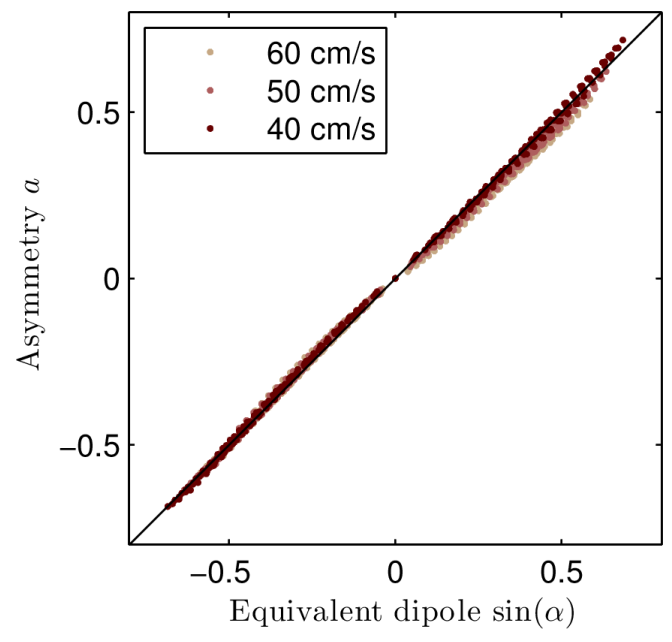

Figure 3. Relation between electrogram asymmetry and sine of the angle of the equivalent dipole. The conduction velocity is color coded. The central point $(0,0)$ corresponds to the absence of epi-endo delay.

tally, we attempted to predict electrogram asymmetry from activation delay $(\delta)$ and transmural coupling $\left(\sigma_{\mathrm{z}}\right)$ using the empirical formula

$$
a \propto \delta \cdot \sigma_{\mathrm{z}}^{p},
$$

where $p$ is an exponent. Figure 4 shows the resulting relations with $p$ adjusted to maximize correlation. In all cases, $p$ was about 0.81 and all correlation coefficients were $>0.99$. The slope, however, depended on conduction velocity, since a narrower range of asymmetries was observed when conduction velocity was higher.

\section{Discussion}

The validity of Eq. (7) relies on the assumption that the distributed current sources within the $2-\mathrm{mm}$ thick tissue can be represented by a line of dipoles. Since the distance between the current sources is of the order of the distance between the sources and the electrode, the critical property of Eq. (7) is that asymmetry does not depend on $z$. This provides more robustness. If $z$ is decreased to $1 \mathrm{~mm}$, the correlation between $a$ and $\sin (\alpha)$ remains $>0.994$. If $z$ becomes larger, at some point, boundary effects come into play.

On the other hand, the formula (4) for the amplitude depends on $z$. For a $2 \mathrm{~mm}$ thickness and a $2 \mathrm{~mm}$ electrodetissue distance, the factor $1 / z$ varies between 0.25 and 0.5 depending on the location of the current source. As a result, the dipole model is less accurate for the amplitude.

The regression using formula (8) showed that, for given conduction properties and all other things being equal, electrogram asymmetry is proportional to the epi-endo delay. Asymmetry is also related to the transmural coupling 


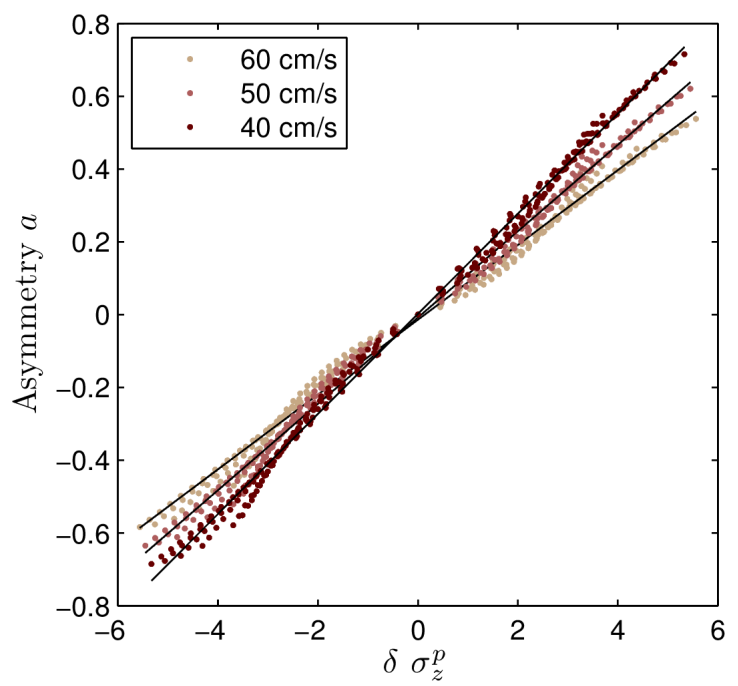

Figure 4. Electrogram asymmetry (endocardial electrode) expressed as a function of delay $(\delta)$ and transmural coupling $\left(\sigma_{\mathrm{z}}\right)$ with an exponent $p=0.80,0.81$ and 0.82 for a conduction velocity of 40,50 and $60 \mathrm{~cm} / \mathrm{s}$ respectively. Linear regression lines are superimposed (black lines).

$\sigma_{\mathrm{z}}$ since the transmural component of the equivalent dipole depends on $\sigma_{\mathrm{z}}$. Note that if $\sigma_{\mathrm{z}}$ is larger, it is more difficult to generate a significant epi-endo delay (Fig. 2).

The design of our model was intended to reproduce in a thin-walled monodomain framework the hypotheses of the line of dipoles. This provides a proof-of-concept in the best-case scenario. In a three-dimensional structure, the combined effects of wavefront curvature, rotating fiber orientation, trabeculated structure and conduction heterogeneity would create confounding factors.

\section{Conclusion}

The orientation of the equivalent dipole is a determinant of electrogram asymmetry that depends on both activation delay and epicardial-endocardial coupling. Further work is needed to understand how these results can be extended in fully three-dimensional models of the atria.

\section{Acknowledgements}

This work was supported by the Natural Sciences and Engineering Research Council of Canada (grant RGPIN-
2015-05658) and by the Mitacs Globalink program.

\section{References}

[1] Villacastin J, Almendral J, Arenal A, Castellano NP, Gonzalez S, Ortiz M, Garcia J, Vallbona B, Moreno J, Portales JF, Torrecilla EG. Usefulness of unipolar electrograms to detect isthmus block after radiofrequency ablation of typical atrial flutter. Circulation 2000;102(25):3080-5.

[2] Houben RPM, de Groot NMS, Smeets JLRM, Becker AE, Lindemans FW, Allessie MA. S-wave predominance of epicardial electrograms during atrial fibrillation in humans: indirect evidence for a role of the thin subepicardial layer. Heart Rhythm 2004;1(6):639-47.

[3] Jacquemet V, Virag N, Ihara Z, Dang L, Blanc O, Zozor S, Vesin JM, Kappenberger L, Henriquez C. Study of unipolar electrogram morphology in a computer model of atrial fibrillation. J Cardiovasc Electrophysiol 2003;14(10 Suppl):S172-9.

[4] Schuessler RB, Kawamoto T, Hand DE, Mitsuno M, Bromberg BI, Cox JL, Boineau JP. Simultaneous epicardial and endocardial activation sequence mapping in the isolated canine right atrium. Circulation 1993;88(1):250-63.

[5] Eckstein J, Maesen B, Linz D, Zeemering S, van Hunnik A, Verheule S, Allessie M, Schotten U. Time course and mechanisms of endo-epicardial electrical dissociation during atrial fibrillation in the goat. Cardiovasc Res 2011;89(4):816-24.

[6] Gharaviri A, Verheule S, Eckstein J, Potse M, Kuijpers NHL, Schotten U. A computer model of endo-epicardial electrical dissociation and transmural conduction during atrial fibrillation. Europace 2012;14 Suppl 5:v10-v16.

[7] Labarthe S, Bayer J, Coudiere Y, Henry J, Cochet H, Jais P, Vigmond E. A bilayer model of human atria: mathematical background, construction, and assessment. Europace 2014; 16 Suppl 4:iv21-iv29.

[8] Courtemanche M, Ramirez RJ, Nattel S. Ionic targets for drug therapy and atrial fibrillation-induced electrical remodeling: insights from a mathematical model. Cardiovasc Res 1999;42(2):477-89.

Address for correspondence:

Vincent Jacquemet

Hôpital du Sacré-Cœur de Montréal, Centre de Recherche

5400 boul. Gouin Ouest

Montreal (Québec) Canada H4J 1C5

vincent.jacquemeteumontreal.ca 